ORIGINAL ARTICLE

\title{
Role of p53, apoptosis, and cell proliferation in early stage Epstein-Barr virus positive and negative gastric carcinomas
}

\author{
H H Ishii, G C Gobe, J Yoneyama, M Mukaide, Y Ebihara
}

J Clin Pathol 2004;57:1306-1311. doi: 10.1136/jcp.2003.015081

See end of article for authors' affiliations

\section{Correspondence to:}

Dr H Ishii, Department of Pathology, Tokyo Medical University, 6-1-1 Shinjuku, Shinjuku-ku, Tokyo 1608402, Japan; hhishii@ tokyo-med.ac.jp

Accepted for publication 16 June 2004

\begin{abstract}
Aims: Mechanisms of Epstein-Barr virus (EBV) associated gastric tumour development are incompletely understood. The interrelations between EBV infection, apoptosis, cell proliferation, and the expression of the tumour suppressor gene p53 was investigated in 133 early stage gastric carcinomas.

Methods: Tumour tissue was compared with paired non-tumour tissue. EBV encoded small RNAs (EBERs) determined EBV status. The apoptotic index (Al) was determined by morphology and verified biochemically. p53 and Ki-67 expression (cell proliferation) was assessed using immunohistochemistry. Results: EBV was detected in $14.3 \%$ of the cases. Cell proliferation did not differ significantly between EBV positive and negative cancers. However, within both these groups, the p53 positive and negative subsets differed significantly (EBV positive group: $76.8 \%$ and $55.3 \%$ were p53 positive or negative cancers, respectively; $p<0.05$; EBV negative group: $65.2 \%$ and $51.7 \%$ were $p 53$ positive or negative, respectively; $\mathrm{p}<0.005$ ). The numbers of $\mathrm{p} 53$ expressing EBV positive and negative cases were significantly different (57.9\% and $82.5 \%$, respectively; $p<0.05)$. Compared with cell proliferation, apoptosis was significantly lower in EBV positive versus negative cancers (AI of 4.36 and 6.50 , respectively; $p<0.01$ ). The p53 positive and negative subsets also differed significantly in Al (EBV positive group: Al of 5.13 and 3.30 for p53 positive and negative cancers, respectively; $p<0.05$ : EBV negative group: Al of 6.84 and 4.90 for p53 positive and negative cancers, respectively; $p<0.05$ ).

Conclusions: These factors probably combine to promote development and progression of early stage gastric carcinomas and, at the same time, ensure the survival of EBV itself.
\end{abstract}

\begin{abstract}
"Little attention has been paid to the role that Epstein-Barr virus infection may have in the tumorigenesis and development of early stage gastric carcinoma"
\end{abstract}

One of the most commonly mutated genes in human cancers is $\mathrm{p} 53 .^{78}$ One of its main functions is the control of cell cycle progression. ${ }^{9}$ Normally, p53 acts at the Gl/S phase of the cell cycle to allow the cell to repair its DNA by causing cell cycle arrest, and at the same time preparing the cell for apoptosis with a G2/M block. If DNA repair is unsuccessful, p53 then acts in the apoptotic pathway. In normal tissue, such changes in the expression of p53 are transient. Apoptosis and cell cycle arrest may be reduced when p53 is mutated, allowing tumour development and progression. The mutated protein is known to be resistant to degradation, and this stabilised protein product is often detected using immunohistochemistry. ${ }^{10}$ There are some limitations to this methodology in that the detection of the stabilised protein does not necessarily indicate altered function, and other analyses or associated markers for function may need to be used.

There is some indication that members of the herpesvirus family, like EBV, modulate or mutate p53 for their own survival. ${ }^{11}$ For example, the cytomegalovirus IE2 protein, the Kaposi's sarcoma associated herpesvirus open reading frame K8 protein, and the human herpesvirus 6 open reading frame 1 protein all prevent degradation of p53, thus increasing its overall concentration but inhibiting its normal actions. ${ }^{12-14}$ The latency associated nuclear antigen of Kaposi's sarcoma associated herpesvirus and latent membrane protein 1 of EBV also interfere with p53 function..$^{15}$

EBV associated gastric cancer is sometimes characterised by a prominent lymphoid infiltration and stroma. ${ }^{17}{ }^{18}$ Kijima and colleagues have recently shown that the infiltration of lymphocytes in EBV positive gastric cancers is not linked to improved prognosis, and that EBV infection itself did not have an effect on the prognosis of patients with gastric cancer. ${ }^{17}$ In our present study, a large group of 133 cases of early gastric carcinoma with little lymphoid infiltration was analysed for EBV infection using EBV encoded RNAs (EBERs) and in situ hybridisation (ISH). EBERs are the most abundant viral transcripts in latently EBV infected cells, and their transfection into EBV negative lymphomas increases resistance to apoptosis. ${ }^{19}$ To define some of the molecular characteristics of the EBV free and EBV associated

Abbreviations: Al, apoptotic index; BCIP-NBT, 5-bromo-4-chloro- 3indolyl-phosphate/nitroblue tetrazolium; EBER, Epstein-Barr virus encoded small RNA; EBV, Epstein-Barr virus; ISH, in situ hybridisation; PBS, phosphate buffered saline; PCR-SSCP, polymerase chain reaction single stranded conformational polymorphism; PNA, peptide nucleic acid; TBS, Tris buffered saline; TUNEL, terminal deoxyribonucleotidyl transferase mediated dUTP-digoxigenin nick end labelling 


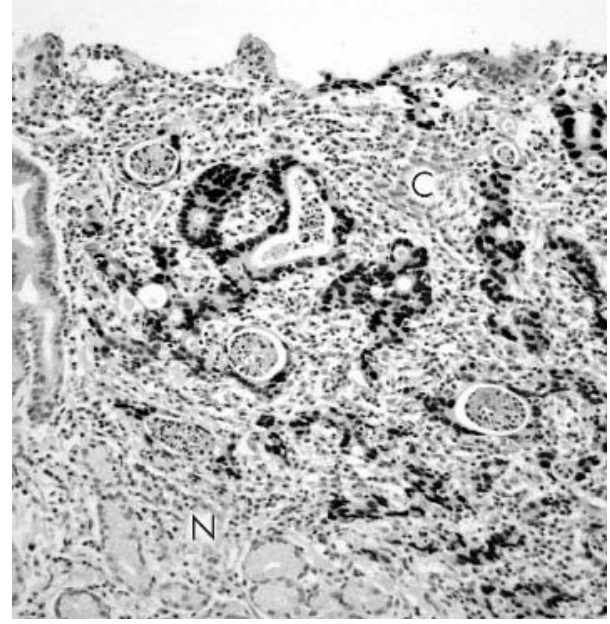

Figure 1 Epstein-Barr virus (EBV) encoded small RNA (EBER) in situ hybridisation. In this example, the nuclei of the carcinoma cells are positive for EBER. At the upper right area of the photomicrograph is a gastric cancer (C) positive for EBV and in the lower left area is an EBV negative non-carcinoma area (N).

forms of these gastric cancers, the association between the expression of p53, cell proliferation, and cell death (apoptosis) was investigated.

\section{MATERIALS AND METHODS \\ Specimens}

One hundred and thirty three gastric adenocarcinomas that had been surgically resected were assessed as early stage cancers (Tl) using the TNM classification of malignant tumours set out by the International Union Against Cancer. ${ }^{20}$ Samples were fixed, embedded in paraffin wax, and sectioned using routine methods. Confirmation as early stage (T1) was made on haematoxylin and eosin stained histological sections, on the basis of depths of invasion using microscopy (mucosa or submucosa). It was also confirmed

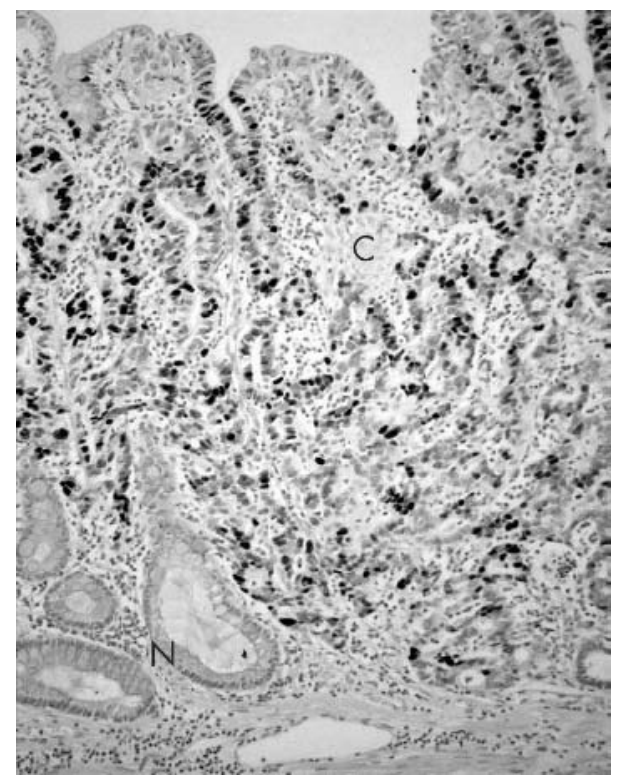

Figure 2 Immunohistochemistry for p53. p53 expression was nuclear and was found in carcinoma cells, but rarely in non-carcinoma cells. C, carcinoma; N, non-carcinoma. histologically that this group had features of gastric cancer without a pronounced degree of lymphocytic infiltration.

\section{EBER in situ hybridisation}

Sections (5-6 $\mu \mathrm{m}$ thick) on glass slides were prepared for ISH with a probe complementary to the two nuclear EBERs encoded by EBV (EBV peptide nucleic acid (PNA) probe; PNA ISH detection kit; Dako, Glostrup, Denmark). In brief, after dewaxing with xylene, and rehydration through alcohols to water, the sections were treated with kit proteinase $\mathrm{K}$ in Tris buffered saline (TBS; pH 7.5) for 20 minutes at room temperature to increase their permeability to the probe. They were then washed with water (three minutes, twice), dehydrated again with 95\% ethanol, and dried at room temperature. Sections were then hybridised with the EBER PNA probe for one hour at $55^{\circ} \mathrm{C}$. The samples were immersed in kit stringent wash solution for 25 minutes at $55^{\circ} \mathrm{C}$ and washed in TBS. They were then conjugated with anti-FITC/ AP (alkaline phosphatase conjugated antibody to fluorescein isothiocyanate) and washed with TBS (for three minutes, three times). BCIP-NBT (5-bromo-4-chloro- 3-indolyl-phosphate/nitroblue tetrazolium) was used as the chromogen for visualising EBER positivity. Negative controls had no EBV PNA probe applied. Positive control tissue was supplied with the kit.

\section{Evaluation of EBV ISH}

After ISH was performed, all specimens were classified as positive or negative according to the presence or absence of detectable BCIP-NBT staining indicative of EBER in the nuclei of the cancer cells.

\section{Immunohistochemistry}

Sections on glass slides were stained for the apoptosis related protein $\mathrm{p} 53$ and the cell proliferation factor Ki-67. The primary antibodies were antihuman p53 antibody (clone DO7; mouse monoclonal IgG2b; diluted $1 / 100$ with phosphate buffered saline (PBS); Dako) and antihuman Ki-67 antibody (clone MIBl; mouse monoclonal IgGl; diluted 1/100 with PBS; Immunotech, Marseille, France). The LSAB staining kit (Dako, Carpinteria, USA) was used. Briefly, after dewaxing the sections with xylene and rehydration through alcohols to PBS, endogenous peroxidase activity was blocked with $0.3 \%$ hydrogen peroxide in methanol. Antigen retrieval was carried out by microwave treating the sections for 15 minutes in $10 \mathrm{mM}$ citrate buffer solution ( $\mathrm{pH} 6.0)$. Primary

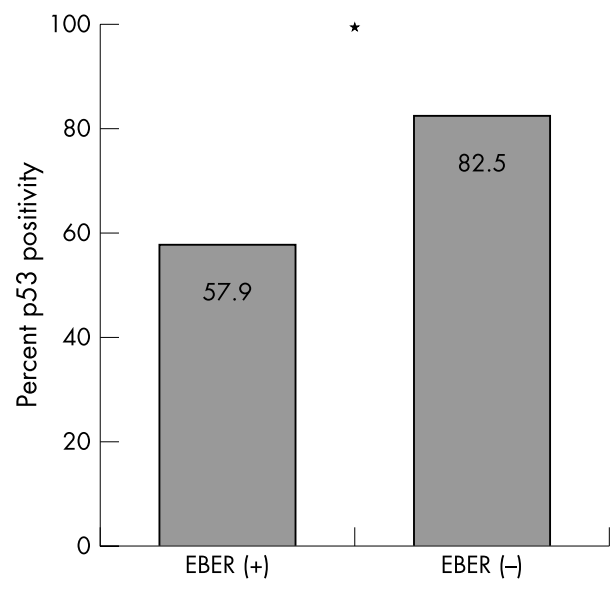

Figure 3 The percentage of $\mathrm{p} 53$ positive (overexpressing) cases: $57.9 \%$ of Epstein-Barr virus (EBV) positive (EBER+) and $82.5 \%$ of $E B V$ negative (EBER-) cases were $\mathrm{p} 53$ positive. The asterisk indicated a significant difference $(p<0.05)$. 
antibodies were incubated overnight at $4^{\circ} \mathrm{C}$. The demonstration of binding sites with the peroxidase reaction was achieved with diaminobenzidine tetrahydrochloride $(0.25 \mathrm{mg}$ dissolved in $1 \mathrm{ml} 0.02 \%$ hydrogen peroxide). PBS was used for rinsing between each step. Sections were lightly counterstained with methyl green solution, sufficient to aid in tissue orientation and recognition but not to influence the judgment of immunohistochemical positivity.

\section{Positivity of immunohistochemistry for p53}

Staining for p53 protein was nuclear, and all cases were examined in the mucosa. At least 20 randomly selected $\times 1000$ microscope fields were observed. The mutant protein is known to be resistant to degradation, and the stabilised protein can easily be detected using immunohistochemistry. ${ }^{10}{ }^{11}$ For assessing p53, each case was finally regarded as overexpressing $\mathrm{p} 53$ protein ( $\mathrm{p} 53$ positive) using the established parameter of over $5 \%$ cellular staining, following methods described previously. ${ }^{21}$ In addition to assessing p53 overexpression in the entire gastric cancer group (133 cases), the EBER positive and negative cases were grouped into subsets that were p53 positive or negative, and these subsets were then analysed for cell proliferation and apoptosis.

\section{Apoptotic index}

At least 2000 cancer cells in the mucosa were screened for apoptosis using the haematoxylin and eosin stained sections and defined morphological criteria (condensed, often crescentic, nuclear chromatin; cell shrinkage and cytoplasmic eosinophilia; cell blebbing; presence of apoptotic bodies). ${ }^{22}$ The percentage of apoptotic cells was calculated. This was recorded as the absolute level of apoptosis. In addition, at least 2000 non-cancer epithelial cells in the mucosa were observed, and the percentage of apoptotic cells was also calculated in the paired non-cancerous tissue section. An apoptotic index (AI) was then calculated as percentage apoptosis in the cancer/non-cancer area. This method has been published, ${ }^{23}$ and it is argued that this index reflects more accurately the relevance of apoptosis in early stage gastric cancers. A terminal deoxyribonucleotidyl transferase mediated dUTP-digoxigenin nick end labelling (TUNEL) apoptosis detection kit (catalogue number, 295-53501;

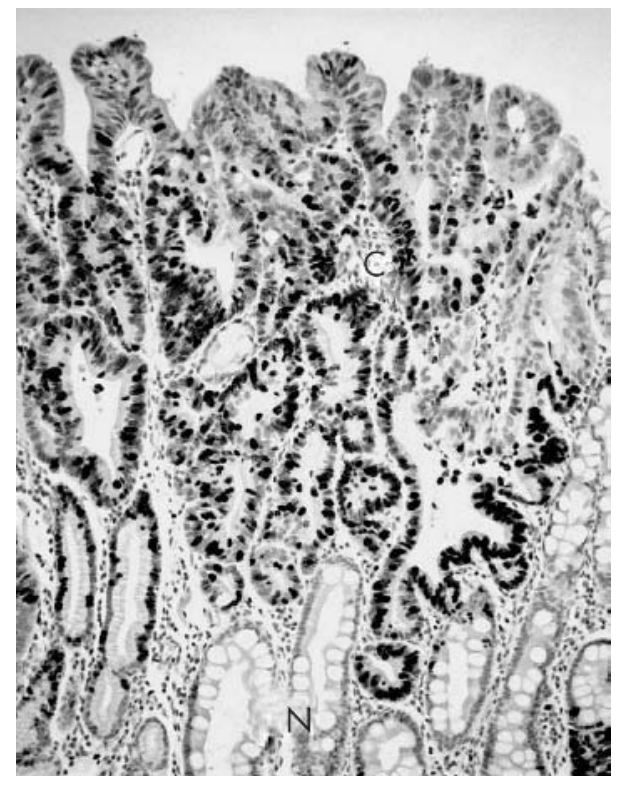

Figure 4 Ki-67 immunohistochemistry. Ki-67 positive nuclei were seen in both carcinoma $(\mathrm{C})$ and non-carcinoma $(\mathrm{N})$ cells, but the proportion was higher in cancer tissue.
Wako, Osaka, Japan) was also used to verify apoptosis biochemically. Morphology was finally selected as the more reliable method for the assessment of apoptosis, as has been suggested previously ${ }^{22}$. Although a good correlation has been reported between apoptosis identified by TUNEL and morphology, ${ }^{24}$ there are now several publications that indicate caution should be used for biochemical methods of apoptosis identification because of the chance of spurious labelling of any cell having DNA strand breakage, a change that may be more common in formalin fixed human specimens. ${ }^{25}$ Necrotic foci in the gastric cancer sections were not assessed.

\section{Cell proliferation}

Staining for the proliferation factor Ki-67 was nuclear. Over 2000 cancer cells in the mucosa were observed in every case, and the percentage positivity was calculated according to published methods. ${ }^{26}$

\section{Statistical analysis}

The relations between each group (mean (SEM)) for the apoptotic and cell proliferation indices were analysed using the Student's two tailed $t$ test. The numbers in the p53 positive (overexpressing) or negative subsets were analysed for different analytical parameters using the $\chi^{2}$ test. For all analyses, $\mathrm{p}$ values of less than 0.05 were considered to be significant.

\section{RESULTS \\ EBER positivity}

Most of EBER positive gastric carcinoma cells showed moderate to strong staining in their nuclei, but some cancer cells in a few cases showed a variation in the intensity. Weakly stained cells were found in close proximity to strongly positive carcinoma cells. Non-cancer cells in the surrounding mucosa were negative for EBERs. EBER positive gastric carcinomas numbered $14.3 \%$ (19 of 133) of the total cases. Figure 1 shows the expression of EBERs in EBV positive gastric cancers versus EBV negative non-carcinoma areas.

\section{p53 overexpression}

Positivity for the p53 protein was nuclear and found in some gastric carcinoma cells, but few non-carcinoma cells (fig 2). p53 positivity (expression $>5 \%$ ) was seen in 11 of 19 $(57.9 \%)$ and 94 of 114 (82.5\%) EBER positive and negative early stage gastric carcinomas, respectively; therefore, positivity was approximately $25 \%$ lower in EBER positive tumours than in negative tumours (fig 3). The $\chi^{2}$ test indicated a significant difference between EBER positive and negative gastric cancers $(\mathrm{p}<0.05)$.

\section{Cell proliferation}

Positivity for Ki-67 was nuclear and seen in both gastric carcinoma cells and non-carcinoma cells. Ki-67 immunopositive nuclei were more frequent in the gastric cancer sections compared with non-cancer tissue (fig 4). The mean (SEM) percentage of Ki-67 positive nuclei was $67.8 \%$ (4.2\%) versus $62.9 \%(1.8 \%)$ in EBER positive versus negative gastric carcinomas (fig 5A) $(\mathrm{p}=0.310295 ;>0.05$ in $t$ test). In EBER positive cancers, mean (SEM) cell proliferation was $76.8 \%(2.4 \%)$ versus $55.3 \%(7.7 \%)$ in p53 positive and negative cancers, respectively (fig 5B; $p=0.029291$; $<0.05$ in $t$ test). In EBER negative cancers, mean (SEM) cell proliferation was $65.2 \%(1.8 \%)$ versus $51.7 \%(5.3 \%)$ in p53 positive and negative cancers, respectively (fig 5B; $\mathrm{p}=0.004728 ;<0.005$ in $t$ test $)$. 

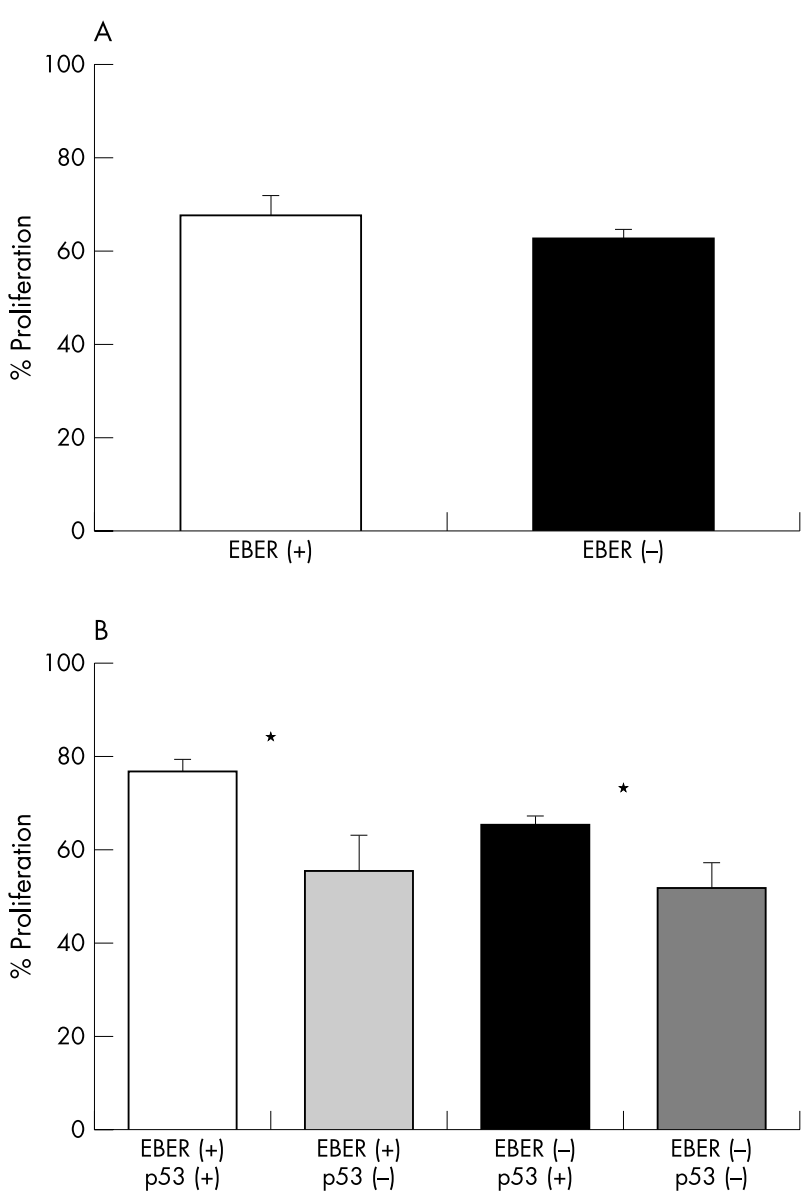

Figure 5 Proliferative indices are shown as the percentage of Ki-67 positivity. (A) Mean (SEM) cell proliferation was $67.8 \%(4.2 \%)$ and $62.9 \%(1.8 \%)$ in Epstein-Barr virus (EBV) positive and negative gastric carcinomas, respectively. There was no significant difference ( $p=0.310295 ; p>0.05$ in $t$ test). (B) Mean (SEM) cell proliferation in EBV positive (EBER+) cancers was $76.8 \%(2.4 \%)$ and $55.3 \%(7.7 \%)$ for p53 positive $(\mathrm{p} 53+)$ or negative ( $\mathrm{p} 53-$ ) cancers, respectively $\left({ }^{*} p=0.029291 ; p<0.05\right.$ in $t$ test). Mean (SEM) cell proliferation in EBV negative (EBER-) cancers was $65.2 \%(1.8 \%)$ and $51.7 \%(5.3 \%)$ for p53 positive ( $\mathrm{p} 53+)$ or negative ( $\mathrm{p} 53-$ ) cancers, respectively $l^{*} p=0.004728 ; p<0.005$ in $t$ test $)$.

\section{Apoptosis}

Apoptosis was identified by cell shrinkage and condensed nuclear chromatin and these were seen in cancer tissue (fig 6). Mean (SEM) AIs were 4.36 (0.50) versus 6.50 (0.54) in EBER positive or negative gastric cancers, respectively (fig 7A; $\mathrm{p}=0.005315,<0.01$ in $t$ test). The $\mathrm{p} 53$ positive and negative subsets of the EBER positive gastric cancers had mean (SEM) AIs of $5.13(0.76)$ versus 3.30 (0.39), respectively (fig $7 \mathrm{~B} ; \mathrm{p}=0.049956 ;<0.05$ in $t$ test). In EBER negative cancers, the $\mathrm{p} 53$ positive and negative subsets had mean (SEM) AIs of $6.84(0.64)$ versus $4.90(0.70)$, respectively (fig 7B; $\mathrm{p}=0.046012 ;<0.05$ in $t$ test).

\section{DISCUSSION}

EBV is a herpesvirus that is carried by more than $90 \%$ of the adult population worldwide. The infection is often nonsymptomatic and non-pathogenic. ${ }^{27}$ After primary infection, the virus persists as a latent infection of resting memory $\mathrm{B}$ cells in the peripheral blood. ${ }^{1}$ However, EBV infection is also involved in the pathogenesis of several tumours. ${ }^{28} \mathrm{EBV}$ proteins have been localised to malignant epithelial cells and dysplastic epithelium, including that found in

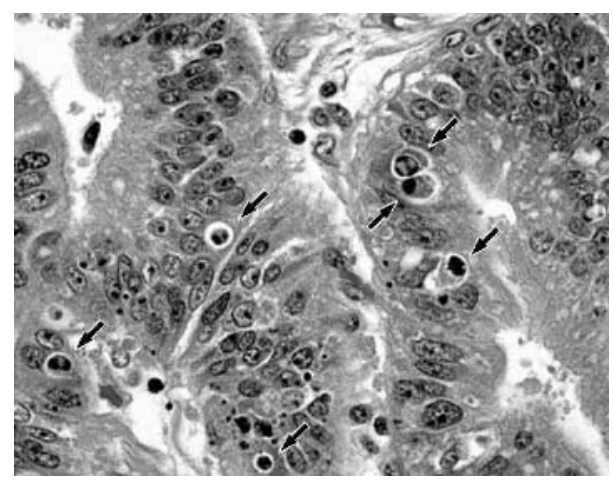

Figure 6 Morphological identification of apoptosis in gastric cancer tissue. The arrows indicate apoptotic cells, many of which have condensed and blebbing nuclei.
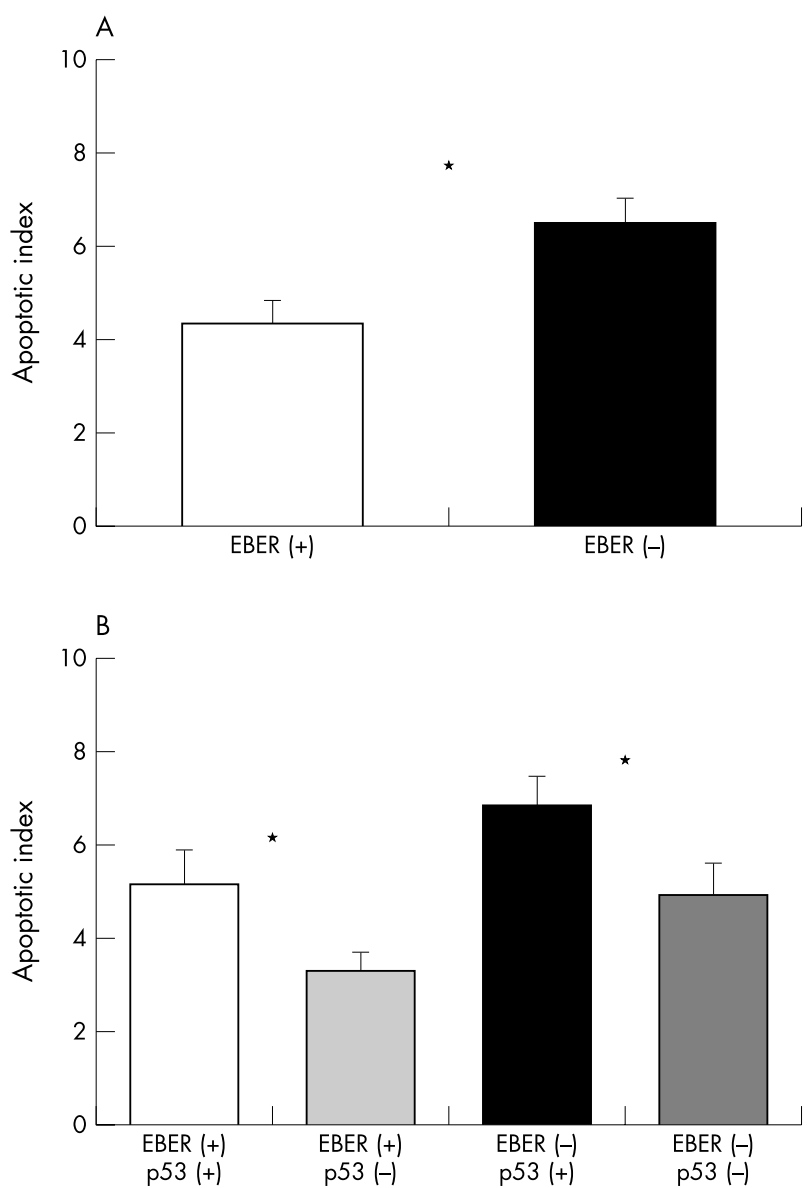

Figure 7 Apoptotic indices. (A) Mean (SEM) apoptotic indices were $4.36(0.50)$ and $6.50(0.54)$ in Epstein-Barr virus (EBV) positive (EBER+) and negative (EBER-) gastric carcinomas, respectively

$\left({ }^{*} p=0.005315 ; p<0.01\right.$ in $t$ test). (B) Mean (SEM) apoptotic indices in EBV positive (EBER+) cancers were $5.13(0.76)$ and $3.30(0.39)$ for p53 positive (p53+) or negative (p53-) cancers, respectively $\left({ }^{*} \mathrm{p}=0.049956 ; \mathrm{p}<0.05\right.$ in $t$ test). Mean (SEM) apoptotic indices in EBV negative (EBER-) cancers were $6.84(0.64)$ and $4.90(0.70)$ for p53 positive ( $\mathrm{p} 53+)$ or negative ( $\mathrm{p} 53-$ ) cancers, respectively ${ }^{*} \mathrm{p}=0.046012 ; \mathrm{p}<0.05$ in $t$ test).

nasopharangeal cancers and gastric carcinomas. ${ }^{11}$ 29-32 Although EBV is known to immortalise cells, giving them the capacity for permanent cell cycling and proliferation, 
another important mechanism that EBV infection uses in the promotion of tumour development is the inhibition of apoptosis. It may do this by modulating the expression of various apoptosis related oncogenes and tumour suppressor genes. Our present study investigated the possible interrelations between EBV infection, p53 overexpression, and levels of apoptosis and cell proliferation, specifically in a large group (133 cancers) of early stage ( $\mathrm{Tl}$ ) gastric carcinomas with little lymphocytic infiltration.

Groups of EBV positive and negative cancers were segregated. Of the total sample, $14.3 \%$ were EBV positive. In this EBV positive group, $57.9 \%$ were $\mathrm{p} 53$ positive compared with $82.5 \%$ in the EBV negative group. The significantly lower proportion of p53 overexpressing cancers in the EBV positive group suggests that EBV may suppress p53 overexpression. There are some other studies that provide direct and indirect support for this theory. ${ }^{33}{ }^{34}$ In vivo, the p53 protein was overexpressed in only four of 23 cases of EBV infected advanced pulmonary carcinoma. ${ }^{33}$ Van Rees and colleagues identified an inverse relation between their immunohistochemical analysis for $\mathrm{p} 53$ protein and EBV positivity in gastric cancers. ${ }^{34}$ One possibility for this result was that EBV interacts with p53 and thereby prevents immunopositivity. Alternatively, the lower prevalence of p53 immunopositivity in the EBV infected group could also indicate a mechanism independent of the p53 pathway. As suggested by van Rees and colleagues, it may be necessary to consider EBV positive carcinomas as having a distinct molecular pathogenesis that does not necessarily follow the dogma of previously defined protein functions. ${ }^{34}$

In our present study, levels of apoptosis were lower in EBV positive gastric carcinomas than in EBV negatives tumours, suggesting that an there may be an association between EBV infection, low levels of apoptosis, and tumour development. The virus may protect itself by minimising apoptosis in its host cells. Much of the evidence for EBV induced resistance to apoptosis comes from work with EBV infected B cells in vitro. For example, EBV infected cells have been found to be resistant to apoptosis in Burkitt's lymphoma cell lines, and also EBV infected B cell lines. ${ }^{45}{ }^{36}$ Our current study also showed that $\mathrm{p} 53$ overexpressing gastric carcinomas undergo apoptosis more than $\mathrm{p} 53$ negative ones in both EBV positive and negative cases. These results suggest that p53 overexpression induces apoptosis, regardless of EBV infection, in this cancer type. The immunohistochemical methodology used in our study has been used previously, and our results concur with those found earlier. For example, Ohfuji and colleagues used the p53 antibody DO7 and reported that p53 positivity was detected more frequently in conventional gastric cancers than in EBV associated ones, and that apoptosis was also seen more frequently. ${ }^{18}$ Other researchers have also found a higher incidence of apoptosis in DO7 positive non-gastric and gastric cancers. ${ }^{37}{ }^{38}$ Wild-type and mutant-type p53 are not distinguished by immunohistochemistry because the DO7 p53 antibody is reactive to both p53 proteins.

\section{"Levels of apoptosis were lower in Epstein-Barr virus (EBV) positive gastric carcinomas than in EBV negatives tumours, suggesting that an there may be an association between EBV infection, low levels of apoptosis, and tumour development ${ }^{\prime \prime}$}

There are certain limitations to the interpretation of results using immunohistochemistry, despite the established argument that only the mutated protein is resistant to rapid degradation, so that this protein should be detected predominantly. However, when investigating gastric
Take home messages

- We investigated a large group of early stage gastric cancers to identify the interrelations between EpsteinBarr virus (EBV) infection, p53 expression, apoptosis, and cell proliferation

- EBV had no apparent effect on proliferation of the host gastric cancer cells, but it was associated with reduced levels of apoptosis

- There were significantly fewer p53 positive cancers in the EBV positive group, suggesting that EBV infection may suppress $\mathrm{p} 53$ overexpression

- These combined factors probably promote the development and progression of early stage gastric cancers, while ensuring the survival of EBV itself

cancers for concordance between p53 expression (measured immunohistochemically) and p53 gene mutations (measured using polymerase chain reaction single stranded conformational polymorphism (PCR-SSCP)), Soong and colleagues found $73 \%$ agreement between the two parameters, ${ }^{21}$ and Hong and colleagues found that $44 \%$ of cancers had p53 immunopositivity and $36 \%$ had p53 mutation using PCRSSCP. ${ }^{39}$ Thus, although the identification of increased concentrations of p53 per se does not confirm function, our results suggest that overexpression of p53 may result in the induction of apoptosis, whereas EBV may suppress p53 to promote its own survival by protecting the host gastric cancer cells against apoptosis.

EBV infection itself was not associated with a significant difference in cell proliferation in early stage gastric carcinomas. However, p53 overexpression, in both EBV positive and negative cancers, did have a positive effect on proliferation, conferring a growth advantage to these early stage gastric carcinomas. These results are in agreement with Aizawa and colleagues, who investigated advanced gastric carcinomas and found a positive correlation between p53 overexpression (immunoreactivity) and proliferation. ${ }^{38}$ In another study, however, a negative correlation was seen between proliferation and the expression of $\mathrm{p} 53$ in both EBV positive and negative cancers. ${ }^{40}$

In conclusion, our investigation used a large group of early stage gastric cancers to identify the interrelations between EBV infection, p53 expression, apoptosis, and cell proliferation. EBV had no apparent effect on proliferation of the host gastric cancer cells, but it was associated with reduced levels of apoptosis. There were significantly fewer p53 positive cancers in the EBV positive group, suggesting that EBV infection may suppress p53 overexpression. It is likely that the combined factors promote the development and progression of early stage gastric cancers and, at the same time, ensure the survival of EBV itself.

\section{ACKNOWLEDGEMENTS}

This study was supported by grants from Tokyo Medical University Cancer Centre and Japan Cytology and Pathology Laboratory.

\section{Authors' affiliations}

H H Ishii, J Yoneyama, Y Ebihara, Department of Pathology, Tokyo Medical University, 6-1-1 Shinjuku, Shinjuku-ku, Tokyo 160-8402, Japan

G C Gobe, Department of Molecular and Cellular Pathology, School of Medicine, University of Queensland, Herston, Brisbane, 4006, Australia M Mukaide, Third Department of Surgery, Tokyo Medical University 


\section{REFERENCES}

1 zur Hausen H. Viruses in human cancers. Science 1991:254:1167-73.

2 Bréchot C, Jaffredo F, Lagorce D, et al. Impact of HBV, HCV and GBV-C/HGV on hepatocellular carcinoma in Europe: results of a European concerted action. J Hepatol 1998;29:173-83.

3 Gregory CD, Dive C, Henderson S, et al. Activation of Epstein-Barr virus latent genes protects human $B$ cells from death by apoptosis. Nature 1991;349:612-14.

4 Ishii H, Tamauchi H, Gobe CG. In the absence of Epstein-Barr virus infection, phorbol ester modulates apoptosis in cycloheximide-treated Burkitt's ymphoma (BJA-B) cells. Int J Exp Pathol 1997;78:123-31.

5 Mauser A, Saito S, Appella E, et al. The Epstein-Barr virus immediate-early protein BZLF1 regulates p53 function through multiple mechanisms. J Virol 2002;76:12503-12.

6 lamaroon A, Pongsiriwet S, Mahanupad P, et al. Oral nonHodgkin lymphomas: studies of EBV and p53 expression. Oral Dis 2003;9:14-8.

7 Lane DP. p53 and human cancers. Br Med Bull 1994;50:582-99.

8 Liu MC, Gelmann EP. p53 gene mutations: case study of a clinical marker for solid tumors. Semin Oncol 2002;29:246-57.

9 Vermeulen K, Berneman ZN, Van Bockstaele DR. Cell cycle and apoptosis. Cell Prolif 2003;36:165-75.

10 Leung SY, Chau KY, Yuen ST, et al. p53 overexpression is different in EpsteinBarr virus-associated and Epstein-Barr virus-negative carcinoma. Histopathology 1998;33:311-17.

11 Moritani S, Sugihara H, Kushima R, et al. Different roles of p53 between Epstein-Barr virus-positive and -negative gastric carcinomas of matched histology. Virchows Arch 2002;440:367-75.

12 Speir E, Modali R, Huang ES, et al. Potential role of human cytomegalovirus and p53 interaction in coronary restenosis. Science 1994;265:391-4.

13 Doniger J, Muralidhar S, Rosenthal $\sqcup$. Human cytomegalovirus and human herpesvirus 6 genes that transform and transactivate. Clin Microbiol Rev 1999;12:367-82.

14 Park J, Seo T, Hwang S, et al. The K-bZIP protein from Kaposi's sarcomaassociated herpesvirus interacts with $\mathrm{p} 53$ and represses its transcriptional activity. J Virol 2000;74:11977-82.

15 Fries KL, Miller WE, Raab-Traub N. Epstein-Barr virus latent membrane protein 1 blocks p53-mediated apoptosis through the induction of the A20 gene. J Virol 1996;70:8653-9.

16 Friborg Jr J, Kong W-p, Hottiger MO, et al. p53 inhibition by the LANA protein of KSHV protects against cell death. Nature 1999:402:889-94.

17 Kijima Y, Ishigami S, Hokita S, et al. The comparison of the prognosis between Epstein-Barr virus (EBV)-positive gastric carcinomas and EBV-negative ones. Cancer Lett 2003;200:33-40.

18 Ohfuji S, Osaki M, Tsujitani S, et al. Low frequency of apoptosis in EpsteinBarr virus-associated gastric carcinoma with lymphoid stroma. Int I Cancer 1996;68:710-15.

19 Komano J, Maruo S, Kurozumi K, et al. Oncogenic role of Epstein-Barr virusencoded RNAs in Burkitt's lymphoma cell line Akata. J Virol 1999:73:9827-31.

20 International Union Against Cancer, Sobin $\mathrm{LH}$, Wittekind $\mathrm{CH}$, eds. TNM classification of malignant tumors, 5th ed. New York: John Wiley \& Sons, 1997.
21 Soong R, Robbins PD, Dix BR, et al. Concordance between p53 protein overexpression and gene mutation in a large series of common human carcinomas. Hum Pathol 1996;27:1050-5.

22 Kerr J, Gobe GC, Winterford CM, et al. Anatomical methods in cell death Methods Cell Biol 1995;46:1-27.

23 Ishii HH, Gobe GC, Pan W, et al. Apoptosis and cell proliferation in the development of gastric carcinomas: associations with c-myc and p53 expression. J Gastroenterol Hepatol 2002;17:966-72.

24 Gobe G, Zhang X-J, Willgoss DA, et al. Relationship between expression of bcl-2 genes and growth factors in ischemic acute renal failure in the rat. J Am Soc Nephrol 2000;1 1:454-67.

25 Grasl-Kraupp B, Ruttkay-Nedecky B, Koudelka H, et al. In situ detection of fragmented DNA (TUNEL assay) fails to discriminate among apoptosis, necrosis, and autolytic cell death: a cautionary note. Hepatology 1995;21:1465-8.

26 Landberg G, Roos G. Proliferating cell nuclear antigen and Ki-67 antigen expression in human haematopoietic cells during growth stimulation and differentiation. Cell Prolif 1993;26:427-37.

27 Cohen Jl. Epstein-Barr virus and the immune system hide and seek. JAMA 1997; 278:510-13

28 Epstein MA. Infection and tumor induction. Nature 1986;321:653-4.

29 Silins SL, Sculley TB. Burkitt's lymphoma cells are resistant to programmed cell death in the presence of the Epstein-Barr virus latent antigen EBNA-4. Int J Cancer 1995:60:65-72.

30 Gulley ML, Pulitzer DR, Eagan PA, et al. Epstein-Barr virus infection is an early event in gastric carcinogenesis and is independent of bcl-2 expression and p53 accumulation. Hum Pathol 1996;27:20-7.

31 Lu Q-L, Elia G, Lucas S, et al. Bcl-2 proto-oncogene expression in EpsteinBarr-virus-associated nasopharyngeal carcinoma. Int J Cancer 1993;53:29-35.

32 Yamamoto N, Tokunaga $M$, Uemura $Y$, et al. Epstein-Barr virus and gastric remnant cancer. Cancer 1994;74:805-9.

33 Chang Y-L, Wu C-T, Shih J-Y, et al. New aspects in clinicopathologic and oncogene studies of 23 pulmonary lymphoepithelioma-like carcinomas. Am J Surg Pathol 2002;26:715-23.

34 van Rees BP, Caspers E, zur Hausen A, et al. Different patterns of allelic loss in Epstein-Barr virus-positive gastric cancer with emphasis on the p53 tumor suppressor pathway. Am J Pathol 2002;161:1207-13.

35 Henderson S, Rowe M, Gregory C, et al. Induction of bcl-2 expression by Epstein-Barr virus latent membrane protein 1 protects infected $B$ cells from programmed cell death. Cell 1991;65:1107-15.

36 Fagard R, Mouas H, Dusanter-Fourt I, et al. Resistance to fludarabine-induced apoptosis in Epstein-Barr virus infected B cells. Oncogene 2002;21:4473-80

37 Hiroshima K, lyoda A, Toyozaki T, et al. Proliferative activity and apoptosis in thymic epithelial neoplasms. Mod Pathol 2002;15:1326-32.

38 Aizawa K, Ueki K, Suzuki S, et al. Apoptosis and bcl-2 expression in gastric carcinomas: correlation with clinicopathological variables, p53 expression cell proliferation and prognosis. Int J Oncol 1999; 14:85-91.

39 Hong S-I, Hong W-S, Jang J-J, et al. Alterations of p53 gene in primary gastric cancer tissue. Anticancer Res 1994;14:1251-5.

40 Renault B, van den Broek M, Fodde $R$, et al. Base transitions are the most frequent genetic change at p53 in gastric cancer. Cancer Res 1993;53:2614-17. 